\title{
Development of On-Line Tritium Monitor Based Upon Artificial Diamond for Fusion Applications
}

\author{
M. Pillon, M. Angelone, P. Batistoni, R. Villari, S. Almaviva, Marco Marinelli, E. Milani, G. Prestopino,
}

C. Verona, and G. Verona Rinati

\begin{abstract}
In this paper a novel on-line tritium monitor is presented. It is made with a single crystal diamond detector (SCD) covered with a thin layer of $\mathrm{LiF} 95 \%$ enriched in ${ }^{6} \mathrm{Li}$. Thermal neutrons impinging on the $\mathrm{LiF}$ layer produce $\alpha$ and $\mathrm{T}$ ions which are detected by the active diamond. The pulse height spectrum shows two separated peaks due to $\alpha$ and T ions respectively. By a proper calibration in a reference thermal flux the number of ${ }^{6} \mathrm{Li}$ atoms and thus the absolute $\mathrm{n}+{ }^{6} \mathrm{Li} \rightarrow \alpha+\mathrm{T}$ reaction rate per unitary flux can be established. Once calibrated the detector can be used to measure the tritium production. Due to the many outstanding properties of diamond this detector could operate in the harsh working conditions of a fusion breeding blanket. A test of this detector was performed at the $14 \mathrm{MeV}$ Frascati Neutron Generator (FNG). The detector was inserted inside a mock-up of the European Helium Cooled Lithium Lead (HCLL) Tritium Blanket Module (TBM), designed to validate the neutronic database for fusion application. The mock-up of the TBM was designed to perform a full set of experiments to validate tritium production code prediction comparing the experimental results with calculations. The measured tritium rates with the Li-Diamond detector are described in this paper. Comparison with calculations is in progress and will be reported in a future paper.
\end{abstract}

Index Terms-Nuclear measurements, particle measurements, radiation detectors, tritium.

\section{INTRODUCTION}

$\mathbf{T}$ HE future fusion reactors will need to self-produce tritium fuel. Tritium will be produced in a breeding blanket containing lithium using the reaction $\mathrm{n}+{ }^{6} \mathrm{Li} \rightarrow \alpha+\mathrm{T}$.

Owing to the harsh working conditions available in a breeding blanket (high temperature, huge neutron and gamma fluxes), the accurate measurement of the tritium production (both reaction rate and inventory) is a difficult task.

The Test Blanket Modules (TBM) program in ITER is a major intermediate step for the development of tritium breeder blan-

Manuscript received June 17, 2009; revised February 07, 2011; accepted March 22, 2011. Date of publication May 05, 2011; date of current version June 15, 2011. This work was supported by the Euratom Communities under the contract of association between EURATOM-ENEA, was carried out within the framework of the European Fusion Development Agreement. The views and opinions expressed herein do not necessarily reflect those of the European Commission.

M. Pillon, M. Angelone, P. Batistoni and R. Villari are with the Associazione EURATOM-ENEA sulla Fusione, Centro Ricerche Frascati, I-00044, Frascati (Roma), Italy (e-mail: mario.pillon@enea.it).

S. Almaviva, M. Marinelli, E. Milani, G. Prestopino, C. Verona and G. Verona Rinati are with Dipartimento di Ingegneria Meccanica, Università di Roma "Tor Vergata," I-00133 (Roma), Italy.

Color versions of one or more of the figures in this paper are available online at http://ieeexplore.ieee.org.

Digital Object Identifier 10.1109/TNS.2011.2134868 kets for fusion reactors. In the frame of the TBMs program in ITER, neutronics tests will be performed, both in DeuteriumDeuterium and Deuterium-Tritium plasmas. The aim is to measure the tritium production rate as well as other nuclear quantities, such as the neutron and gamma ray fluence and the nuclear heating, during ITER operations.

For this purpose, the TBMs will be equipped with detectors able to operate with the required accuracy in the fusion breeding blanket conditions, i.e. at high temperature, high magnetic field, and high radiation flux.

In the frame of the European program for the development of the fusion breeder blankets, an activity has been launched for the development of suitable detectors to be used in neutronics tests in the TBMs of ITER, including tritium detectors. Li-Diamond detectors can be good candidates for such application due to the many outstanding properties of diamond, i.e. high temperature operation and radiation hardness.

In this paper we report about a novel on-line tritium production monitor based on a Single Crystal Diamond (SCD) detector used inside a TBM mock-up and about its first application in the mock-up of the Helium Cooled Lithium Lead (HCLL) blanket for ITER. The mock-up featuring all the relevant nuclear details of the ITER-TBM configuration was designed in such a way to reproduce as close as possible the neutron energy spectra occurring in the TBM.

This mock-up has been irradiated by a dedicated $14 \mathrm{MeV}$ neutron source, the Frascati Neutron Generator (FNG) [1]. The measurements obtained with the SCD detector will be compared with Monte Carlo neutron transport calculation performed using a qualified computer code and cross section data base. The calculations are in progress and comparison with the measurements described here will be reported in a future paper.

\section{Diamond Tritium Monitor Construction}

The tritium monitor consists of a multilayered structure obtained by a two step deposition process. A conductive boron doped diamond homoepitaxial layer with approximately $5 \Omega *$ $\mathrm{cm}$ resistivity, used as a backing contact, is deposited, at first, by Microwave Plasma Enhanced CVD (MWPECVD) on a commercial low-cost synthetic HPHT type Ib single crystal diamond substrate, $4 \times 4 \times 0.5 \mathrm{~mm}^{3}$ in size. After that an intrinsic diamond layer is homoepitaxially grown on the doped one, which operates as detecting region. Its thickness can vary from a few microns up to more than 200 microns, depending upon the use of the detector; in our case, since the penetration depth in diamond of the $2.06 \mathrm{MeV} \alpha$-particles and the $2.73 \mathrm{keV}^{3} \mathrm{H}$ ions produced by the $\mathrm{n}+{ }^{6} \mathrm{Li} \rightarrow \alpha+\mathrm{T}$ reaction is only few microns, a thickness of $25 \mu \mathrm{m}$ was chosen. The diamond layer 


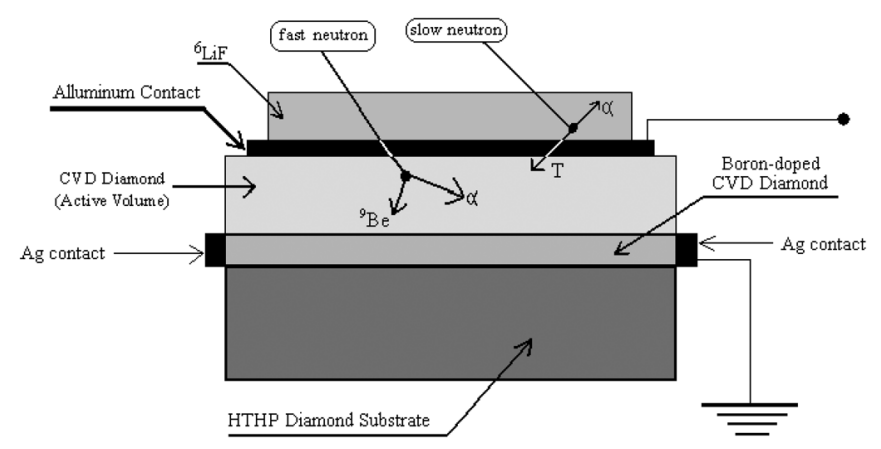

Fig. 1. Sketch of the diamond tritium monitor layout.

has been oxidized, after the growth, by isothermal annealing, at $500{ }^{\circ} \mathrm{C}$, for $1 \mathrm{~h}$ in air, in order to remove the $\mathrm{H}_{2}$ surface conductive layer. Later, a circular aluminum electrode, about $3 \mathrm{~mm}$ diameter and $100 \mathrm{~nm}$ thick is deposited on the diamond surface by thermal evaporation, while annealed silver paint was utilized in order to provide an ohmic contact with the B-doped layer. A $\mathrm{LiF}\left(95 \%{ }^{6} \mathrm{Li}\right.$ enriched) layer, $3 \mu \mathrm{m}$ thick, is finally deposited by vacuum thermal evaporation process on the top of the $\mathrm{Al}$ contact. In such device, $14 \mathrm{MeV}$ neutrons are detected directly in the bulk of the intrinsic diamond layer through the ${ }^{12} \mathrm{C}(\mathrm{n}, \alpha){ }^{9} \mathrm{Be}$ reaction. The produced ${ }^{9} \mathrm{Be}+\alpha$ ions have a total energy $E_{\alpha+B e}=E_{n}-5.7 \mathrm{MeV}$, where $E_{n}$ is the energy of the impinging neutron. Slowed down neutrons are detected through several other reaction mechanisms and produce a continuum in the pulse height spectrum [2]. Sensitivity to thermal neutrons and thus tritium counting is achieved by the $95 \%$ enriched ${ }^{6} \mathrm{LiF}$ layer. Tritium ions and $\alpha$-particles are emitted in opposite directions, so that either of them, but not both, are detected. Fig. 1 shows a sketch of the tritium monitor with the indication of main particle reactions. A more accurate description of such Li-Diamond devices in term of characteristic and performances can be found in [3].

\section{Detector CALibration}

Before starting the measurements in the TBM mock-up the Li-Diamond detector has been calibrated in order to determine its absolute efficiency in term of tritium count rate vs. neutron flux. The calibration was performed using in "ad hoc" thermal neutron irradiation facility at ENEA Bologna designed for the calibration of neutron dosimeters. It consists of a $1 \mathrm{~m} \times 1 \mathrm{~m} \times$ $1 \mathrm{~m}$ polyethylene cube containing three ${ }^{241} \mathrm{Am}-$ Be sources of about $185 \mathrm{GBq}$ each (Fig. 2). The cube contains three co-axial cylindrical cavities of different dimensions where the neutron flux and spectra have been accurately determined. Details of the facility are in [4].

The Li-Diamond was exposed at the neutron flux in one of the cavities. A standard electronic chain consisting in a charge preamplifier, shaping spectroscopy amplifier and a Multichannel pulse-height Analyzer (MCA) was employed.

A pulse-height spectrum (PHS) was acquired for several thousand of seconds since the neutron flux in the used cavity of the irradiation facility is $761 \pm 1.7 \% \mathrm{n} \mathrm{cm}^{-2} \mathrm{~s}^{-1}$. The collected PHS is shown in Fig. 3.

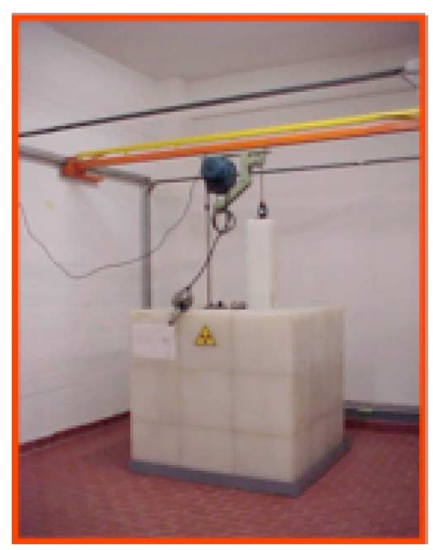

Fig. 2. The thermal neutron irradiation facility.

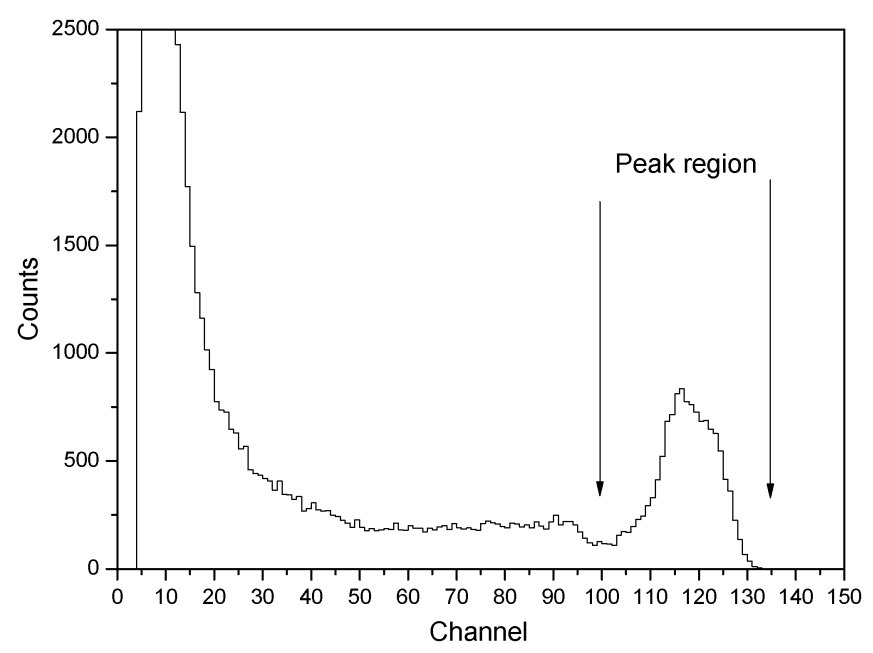

Fig. 3. PHS collected in the thermal neutron facility showing the well define tritium peak produced from ${ }^{6} \mathrm{Li}(\mathbf{n}, \alpha) \mathrm{T}$ reaction.

From the measurement of the counts in the tritium peak $\mathrm{C}_{\mathrm{T}}$, the reaction cross section $\sigma$ and the absolute neutron fluence $\Phi$, the number of reacting ${ }^{6} \mathrm{Li}$ atoms $\mathrm{N}_{6 \mathrm{Li}}$ in the Li-Diamond was determined using the formula (1)

$$
N_{6 L i}=\frac{C_{T}}{\sigma \phi} .
$$

A value of 1324 barn was used for the effective cross section of the ${ }^{6} \mathrm{Li}(\mathrm{n}, \alpha) \mathrm{T}$ reaction in the spectrum of the irradiation facility. $\mathrm{N}_{6 \mathrm{Li}}$ results to be $2.13 \times 10^{17}$ with a $3.7 \%$ uncertainty. The error was derived combining the uncertainty of the neutron flux with the counts statistical error.

\section{EXPERIMENTAL SET-UP}

As described in the introduction this device was used to measure the TPR inside the mock-up of ITER HCLL TBM. The mock-up is made of $\mathrm{Li}-\mathrm{Pb}$ bricks. The $\mathrm{Pb}-\mathrm{Li}$ is of the same type planned to be used in ITER. A photo of the mock-up is shown in Fig. 4 while an accurate description of mock-up characteristics will be reported elsewhere [5].

The central channel of the mock-up was filled with other PbLi bricks, one of them contain the housing for the diamond tritium monitor (Fig. 5). 


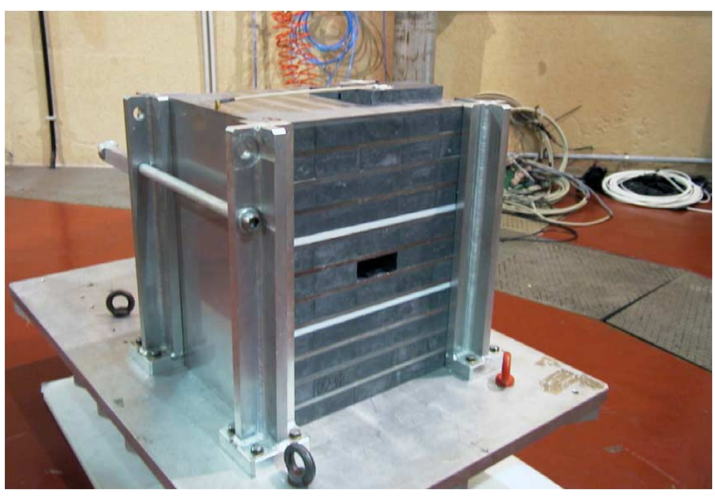

Fig. 4. Photo of the TBM mock-up.

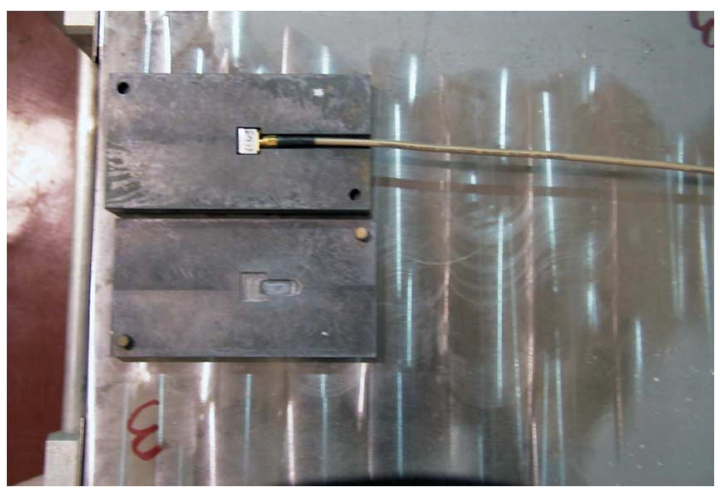

Fig. 5. The lithium-lead brick containing the housing for the tritium monitor

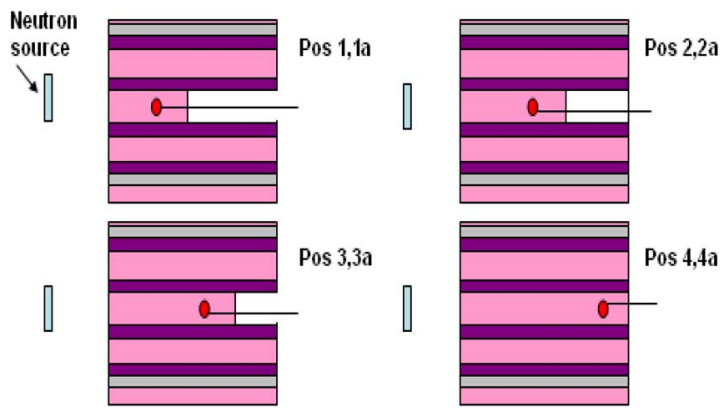

Fig. 6. Sketch of the tritium monitor positions inside the mock-up.

The measurements were performed at four depths inside the block up to last position at $26 \mathrm{~cm}$. Then the measurements were repeated moving in the backward direction. This was done in order to determine an overall uncertainty introduced by the detector positioning since the movement of the brick housing the detector was not easy due to some friction with the central channel of the block.

For a better understanding Fig. 6 shows a sketch of the four monitor positions in the central part of the TBM mock-up.

\section{RESULTS}

During the measurements inside the TBM mock-up the tritium monitor was connected to the same electronic used during the calibration. The mock-up was exposed at eight irradiations

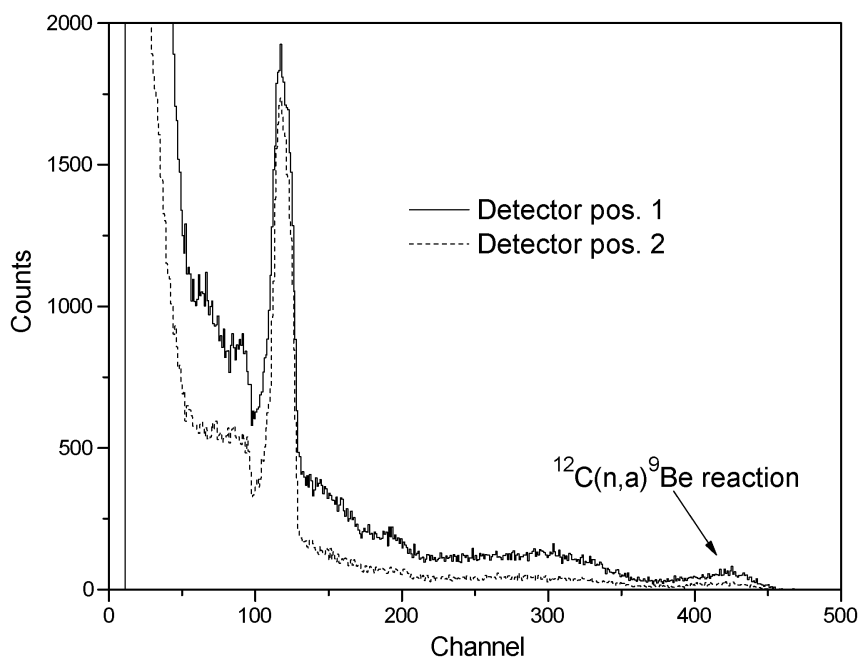

Fig. 7. Detector pulse height spectrum at positions 1 and 2 inside the TBM block.

of the $14 \mathrm{MeV}$ neutrons produced by FNG. The distance of the front block surface from FNG neutron source was $5 \mathrm{~cm}$.

The neutron spectrum inside the mock-up is more complicated than that of the thermal neutron irradiation facility used for the calibration. It contains a fast neutron component which tends to decrease with the depth inside the block.

The Li-Diamond detector has an energy resolution which permits the recognition of the fast neutron component and in particular the presence of neutron able to induce the ${ }^{12} \mathrm{C}(\mathrm{n}, \alpha){ }^{9} \mathrm{Be}$ reaction $(\mathrm{E}>5.7 \mathrm{MeV})$.

This is shown in Fig. 7 where the measured PHS for the Li-Diamond detector in position 1 and 2 are shown.

In Table I are reported the main parameters of the irradiation and the measured Tritium Production Rate as a function of the irradiation positions. The detector positions inside the block, the irradiation time, the source yield and the count rate of the tritium peak in the measured pulse height spectrum are gathered in the table.

The measurements will be compared with calculations which are in progress. Details about the calculations will be reported elsewhere [5].

In this paper the results of the measurements are reported. The measured TPRs moving the detector forward and backward have been normalized to the source yield (multiplied by $1.0 \times$ $10^{10}$ ) and they are gathered in Fig. 8.

The error bars of the experimental points include the statistical uncertainties of the measured count rates and the uncertainty in the source yields determination $( \pm 3 \%)$.

The uncertainty introduced by the detector positioning can be evaluated from Fig. 8. Only in the first measured position there is an error due to detector positioning greater then the uncertainty of the measurement at one standard deviation.

\section{CONCLuSIONS}

The experiment carried out has shown the possibility to measure on-line the TPR using a small detector inside a mock-up of a breeding blanket irradiated with fusion neutrons. The detector 
TABLE I

IRRADIATION PARAMETERS AND MEASURED TPR

\begin{tabular}{|c|c|c|c|c|c|c|c|c|}
\hline & $\begin{array}{c}\text { Pos } \\
1\end{array}$ & $\begin{array}{c}\text { Pos } \\
2\end{array}$ & $\begin{array}{c}\text { Pos } \\
3\end{array}$ & $\begin{array}{c}\text { Pos } \\
4\end{array}$ & $\begin{array}{c}\text { Pos } \\
1 \mathrm{a}\end{array}$ & Pos $2 \mathrm{~b}$ & $\begin{array}{c}\text { Pos } \\
3 \mathrm{~b}\end{array}$ & $\begin{array}{c}\text { Pos } \\
4 \mathrm{~b}\end{array}$ \\
\hline $\begin{array}{c}\text { Depth } \\
(\mathrm{cm})\end{array}$ & 9.2 & 14.8 & 20.4 & 26.0 & 9.2 & 14.8 & 20.4 & 26.0 \\
\hline Tirr (s) & 640 & 631 & 800 & 1221 & 490 & 510 & 651 & 1020 \\
\hline $\begin{array}{c}\text { Source } \\
\text { Yield } \\
\left(10^{10} \mathrm{n} / \mathrm{s}\right)\end{array}$ & 3.80 & 4.05 & 4.06 & 3.86 & 4.68 & 4.90 & 4.79 & 4.40 \\
\hline Meas. T/s & 17.0 & 15.6 & 12.5 & 8.7 & 23.0 & 19.3 & 14.8 & 9.7 \\
\hline
\end{tabular}

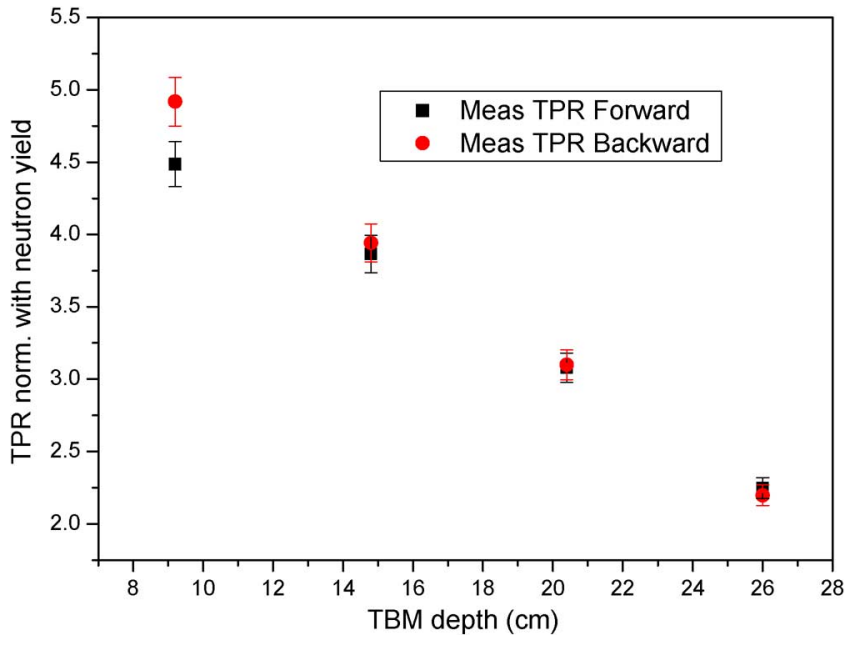

Fig. 8. Comparison of TBR measurements moving the detector forward and backward.

made with a combination of lithium and diamond has shown a reliable operation and the possibility to be accurately calibrated in order to obtain absolute measurements. The preliminary results have shown reliable and stable detector operation at different neutron flux value.

These results are not sufficient to prove that Li-Diamond detector can be used as a reliable on-line tritium monitor in real fusion blanket because in our experiment there were not all the conditions that will be found in a real fusion breeding blanket, i.e. high temperature, magnetic field, high radiation flux. However due to the many outstanding properties of diamond, i.e. high temperature operation, $14 \mathrm{MeV}$ neutrons radiation hardness [6], etc., this type of detector is a good candidate and it will be further developed for this purpose.

\section{REFERENCES}

[1] M. Martone, M. Angelone, and M. Pillon, "The $14 \mathrm{MeV}$ Frascati neutron generator," J. Nucl. Mater., vol. 1661, pp. 212-215, 1994.

[2] M. Pillon, M. Angelone, and A. V. Krasilnikov, "14 MeV neutron spectra measurement with $4 \%$ energy resolution using type IIa diamond device," Nucl. Instrum. Meth. Phys. Res., vol. B101, pp. 473-483, 1995.

[3] S. Almaviva, M. Marinelli, E. Milani, G. Prestopino, A. Tucciarone, C. Verona, G. Verona-Rinati, M. Angelone, D. Lattanzi, M. Pillon, R. M. Montereali, and M. A. Vincenti, "Thermal and fast neutron detection in chemical vapor deposition single-crystal diamond detectors," J. Appl. Phys., vol. 103, p. 054501, 2008.

[4] R. Bedoni, G. Gualdrini, and F. Monteventi, "Developing a thermal neutron irradiation system for the calibration of personal dosemeters in terms of Hp(10)," Rad. Prot. Dos., vol. 110, no. 1-4, pp. 43-48, 2004.

[5] P. Batistoni, M. Angelone, P. Carconi, M. Pillon, K. Kondo, A. Klix, W. Pohorecki, U. Fischer, D. Leichtle, L. Petrizzi, R. Villari, and I. Kodeli, "Neutronics experiments on HCPB and HCLL TBM mock-ups and considerations/strategies for nuclear measurements in ITER," presented at the 9th Int. Symp. on Fusion Nuclear Technology (ISFNT9), Dalian, China, Oct. 11-16, 2009.

[6] M. Pillon, M. Angelone, G. Aielli, S. Almaviva, M. Marinelli, E. Milani, G. Prestopino, A. Tucciarone, C. Verona, and G. Verona-Rinati, "Radiation tolerance of a high quality synthetic single crystal chemical vapor deposition diamond detector irradiated by $14.8 \mathrm{MeV}$ neutrons," J. Appl. Phys., vol. 104, p. 054513, 2008. 